\title{
Growth kinetics of racemic heptahelicene-2-carboxylic acid nanowires on calcite (104)
}

\author{
Mario Einax, ${ }^{1,2, a)}$ Tobias Richter, ${ }^{3}$ Markus Nimmrich, ${ }^{4}$ Philipp Rahe, ${ }^{4, b)}$ Irena G. Stará, ${ }^{5}$ \\ Ivo Starý, ${ }^{5}$ Angelika Kühnle, ${ }^{4}$ and Philipp Maass ${ }^{2, \mathrm{c})}$ \\ ${ }^{1}$ School of Chemistry, Tel Aviv University, Tel Aviv 69978, Israel \\ ${ }^{2}$ Fachbereich Physik, Universität Osnabrück, Barbarastraße 7, 49076 Osnabrück, Germany \\ ${ }^{3}$ Institut für Computerphysik, Universität Stuttgart, Pfaffenwaldring 27, 70569 Stuttgart, Germany \\ ${ }^{4}$ Institut für Physikalische Chemie, Johannes Gutenberg-Universität Mainz, 55099 Mainz, Germany \\ ${ }^{5}$ Institute of Organic Chemistry and Biochemistry, Academy of Sciences of the Czech Republic, v.v.i., \\ Flemingovo nám. 2, 16610 Prague 6, Czech Republic
}

(Received 17 May 2016; accepted 15 September 2016; published online 3 October 2016)

\begin{abstract}
Molecular self-assembly of racemic heptahelicene-2-carboxylic acid on a dielectric substrate at room temperature can be used to generate wire-like organic nanostructures consisting of single and double molecular rows. By means of non-contact atomic force microscopy, we investigate the growth of the wire-like pattern after deposition by experimental and theoretical means. From analyzing the time dependence of the mean row length, two distinct regimes were found. At the early post-deposition stage, the mean length grows in time. Subsequently, a crossover to a second regime is observed, where the mean row length remains nearly constant. We explain these findings by a mean-field rate equation approach providing a comprehensive picture of the growth kinetics. As a result, we demonstrate that the crossover between the two distinct regimes is accomplished by vanishing of the homochiral single rows. At later stages only heterochiral double row structures remain. Published by AIP Publishing. [http://dx.doi.org/10.1063/1.4963724]
\end{abstract}

\section{INTRODUCTION}

The search for new concepts for building electronic devices goes along with the technological challenge to achieve significantly smaller structures down to the atomic scale. In new approaches for device fabrication, promising material systems especially include organic building blocks. Consequently, current research is concerned with the development of functional molecular entities such as molecular transistors or molecular diodes as well as passive elements such as nanowires. ${ }^{1-5}$

Investigating organic materials leading to one-dimensional row growth emerges as a natural necessity. Furthermore, and most importantly, these structures need to be fabricated on nonconducting rather than conducting substrates, to decouple the electronic structure from the underlying support. In this context, self-assembly has gained great importance as a powerful strategy to provide tailored structure formation (for a review, see Ref. 6 and references therein). In order to produce wire-like structures in a predictable manner, it is crucial to elucidate the key underlying processes and their interplay during the growth and ripening in the deposition and post-deposition regime, respectively.

Only few systems forming molecular rows on flat terraces of insulator surfaces, i.e., without the need of nucleation sites such as step edges, are known so far. One example is the formation of one-dimensional rows of copper

\footnotetext{
$\overline{\text { a)Electronic mail: meinax @al.tau.ac.il }}$

b) Now at School of Physics and Astronomy, The University of Nottingham, University Park, Nottingham NG7 2RD, United Kingdom.

c)Electronic mail: maass@uos.de
}

phthalocyanine parallel to the monatomic steps on hydrogen (H) passivated vicinal silicon (111) surfaces. $^{7}$ Another example is the double row formation of hydroxybenzoic acid on calcite (104), ${ }^{8}$ where an ordering of the rows was seen by long-range repulsive interactions, which were supposed to arise from a locally induced substrate-molecule charge transfer.

In recent experiments using non-contact atomic force microscopy (NC-AFM) under ultra-high vacuum (UHV) conditions operated at room temperature, non-planar aromatic molecules have been observed to self-assemble into nanowirelike aggregates on an insulating, nearly defect-free surface. ${ }^{9-11}$ These wire-like row structures of well-defined width were observed after the deposition of racemic heptahelicene-2carboxylic acid ([7]HCA) on the calcite (104) surface. The rows grow on flat terraces and are well-aligned along the [010] substrate direction. From an interplay between NCAFM results and corresponding density functional theory (DFT) calculations, it has been demonstrated that the [7]HCA molecules form heterochiral pairs by hydrogen bonding between the carboxylic groups, while $\pi-\pi$ stacking results in the unidirectional growth of the molecular rows.

In this work, we focus on the growth kinetics of the row formation using a description based on meanfield rate equations. In the past, mean-field rate equation treatments ${ }^{12,13}$ of submonolayer growth have proven to be a powerful approach for modelling the growth kinetics of nanostructure formation for a large variety of material systems (for reviews, see Refs. 19-22). These include metallic ${ }^{23-25}$ and organic adsorbates. ${ }^{26-30}$ Analyses based on the rate 
equation approach can be used to extract fundamental material parameters, such as diffusion barriers and effective binding energies, ${ }^{19,20}$ even for more complex multicomponent systems. $^{22,31-33}$

High-resolution NC-AFM measurements reveal both, single and double row structures of racemic [7]HCA on the calcite (104) surface shortly after the deposition, while the molecular pattern exclusively consists of molecular double rows at later stages. Time-dependent changes of these row patterns are analyzed using the mean length of the grown rows as the observable. Interestingly, two distinct regimes are found from this analysis. This finding and further details of the nanostructure formation are summarized in Sec. II. To understand the growth kinetics, we develop in Sec. III a rate equation theory for the row formation of [7] HCA molecules on the calcite (104) surface.

\section{EXPERIMENTAL SETUP AND OBSERVATIONS}

\section{A. Experimental setup}

The non-planar aromatics used in this study are specifically synthesized racemic [7]HCA molecules, see Fig. 1(a) for molecular models. Details about the synthesis can be found in Ref. 10. The molecules are formed by seven fused benzene rings with one of the ends functionalized by a carboxylic group. Two enantiomers of [7]HCA exist due to the helical form; herein we use a 1:1 racemic mixture of $(M)$ and $(P)$ enantiomers. From the chemical structure, two different intermolecular interactions can be expected. First, the carboxylic groups allow for the formation of intermolecular hydrogen bonds, while the aromatic system of the seven fused benzene rings favours $\pi-\pi$ stacking. Due to the anisotropic molecular structure, these bonds are highly directed.

Calcite (104) surfaces are prepared by cleaving and annealing crystals of optical quality under UHV conditions. ${ }^{14}$ By this method, clean substrates with large terraces and low defect density are formed that allow for imaging with atomic-resolution using NC-AFM. ${ }^{15}$ The [7] HCA molecules are deposited in situ at a flux of $F \simeq 8.3 \times 10^{-5} \mathrm{~s}^{-1} \mathrm{~nm}^{-2}$ for $10 \mathrm{~min}$, leading to substrate coverages of about $\Theta$ $=0.06$ monolayers $(\mathrm{ML}) .^{16}$

The calcite sample is held at room temperature during the deposition. The samples are subsequently imaged using a VT AFM 25 (Omicron Nanotechnology GmbH) operated in the frequency-modulated AFM mode where the tipsample height is adjusted by keeping the frequency shift of an oscillating cantilever at a set point relative to its eigenfrequency. ${ }^{17}$ The frequency shift is related to the tipsample interaction forces ${ }^{18}$ and allows for mapping the surface structure. For clarity, we show herein the frequency shift $\Delta f$ channel; further details about the experimental methods are available in Ref. 9.

\section{B. Experimental findings}

Figure 1(b) presents NC-AFM data of a racemic [7]HCAcovered calcite surface about $6 \mathrm{~h}$ after finishing the deposition. (a)

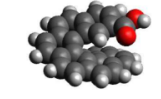

(M)-[7]HCA

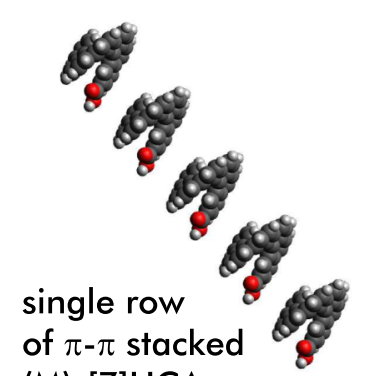

(M)-[7]HCA
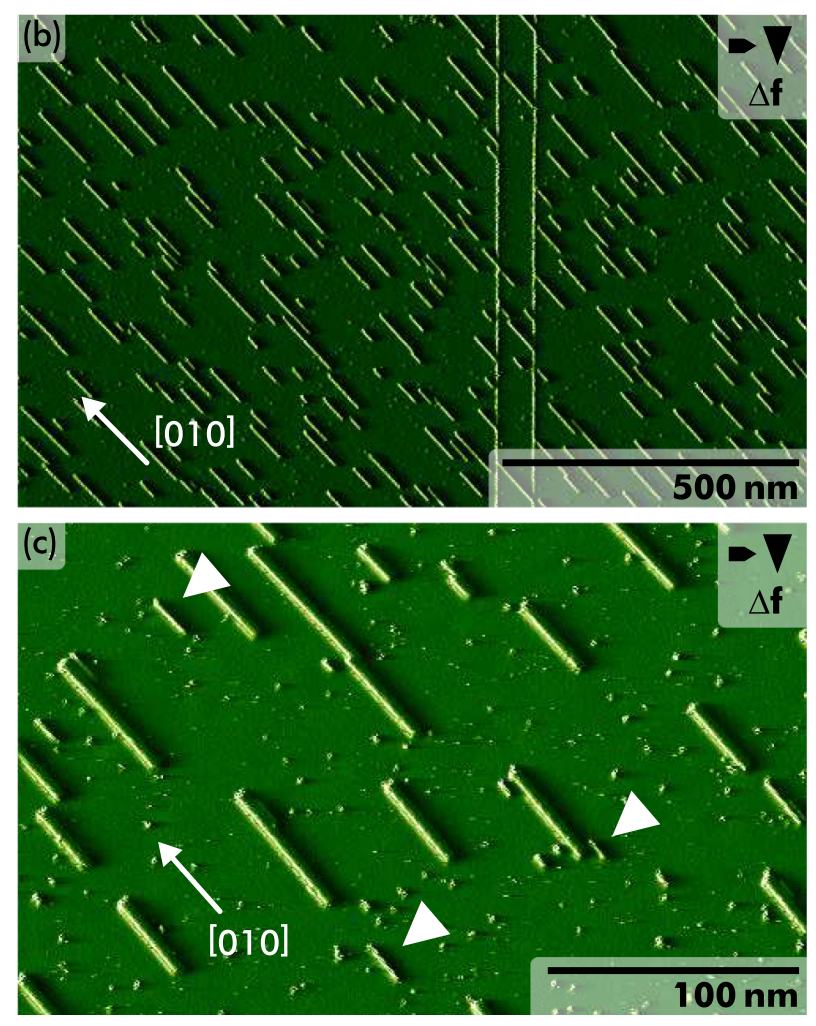

FIG. 1. (a) Structures of the chiral [7]HCA monomers, $\pi-\pi$ bonded homochiral single row, and heterochiral double row formed by H-bonded dimers. ${ }^{9}$ ((b),(c)) NC-AFM images of the (104) calcite surface (b) $6 \mathrm{~h}$ and (c) about $65 \mathrm{~min}$ after finishing the deposition of racemic [7]HCA. ${ }^{9}$ The coverage is $\Theta=0.06 \mathrm{ML}$. Single rows are indicated by arrows in (c).

Wire-like structures of well-defined width formed by the [7]HCA molecules are clearly resolved on flat terraces. Interestingly, no nucleation sites such as step edges are required to form these self-assembled, one-dimensional rows and all rows are well-aligned along the [010] crystallographic direction of the calcite surface. High-resolution images taken on a different sample about 65 min after deposition reveal two distinct row types [see Fig. 1(c)].

The broader rows are of approximately twice the width of the thin ones, suggesting that the thin (broad) rows consist of single (pairs of) [7]HCA molecules, respectively. Detailed structure information was obtained from $a b$ initio DFT calculations, investigating structures composed of homochiral 

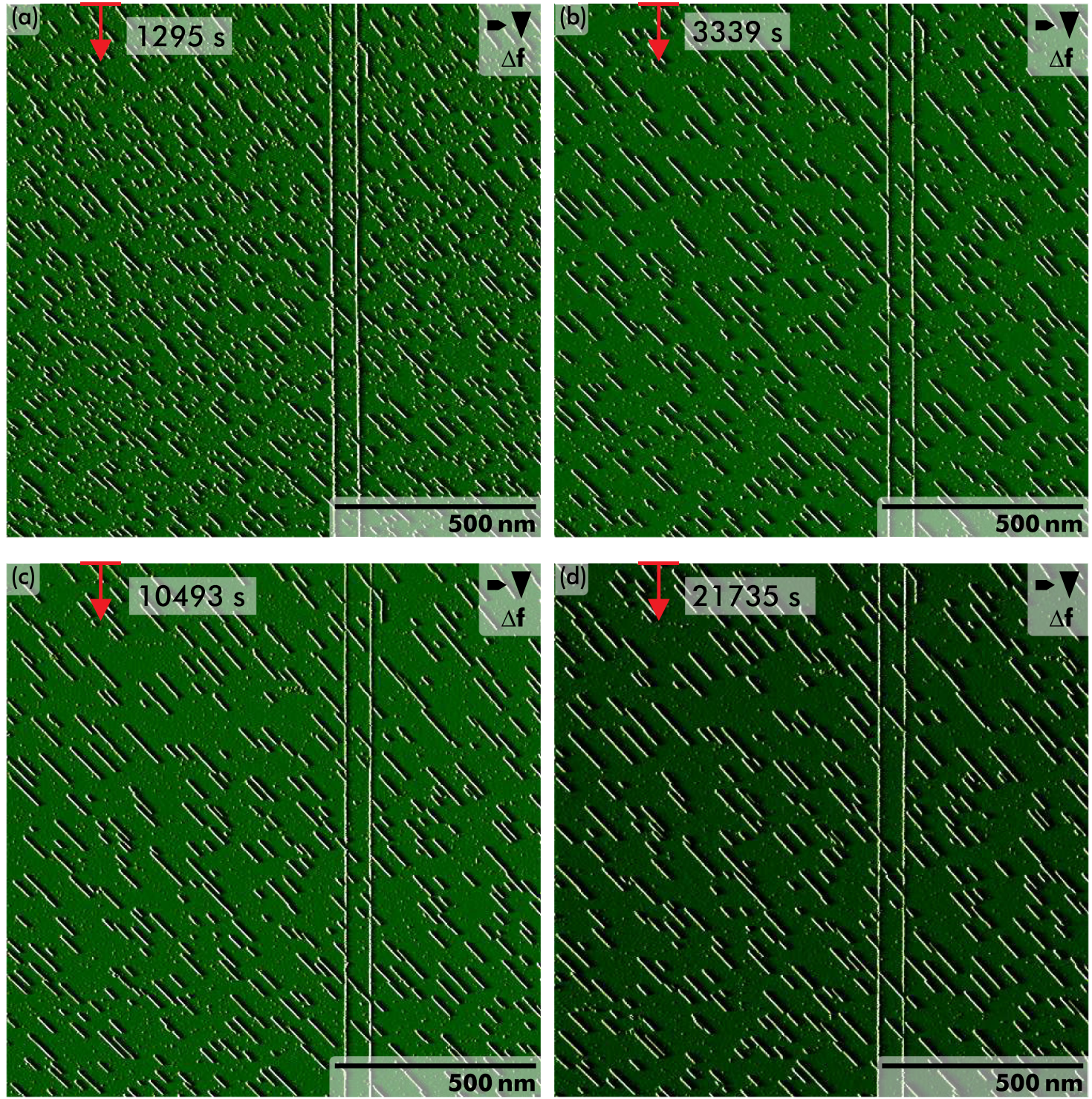

FIG. 2. (a)-(d) NC-AFM images of the ripening process of wire-like racemic [7]HCA nanostructures on defect-free (104) calcite terraces at four different scanning times for $\Theta=0.06 \mathrm{ML}$. The red arrows mark the starting positions of the scans.

single as well as homochiral double and heterochiral double rows. ${ }^{9}$ These calculations suggest a structural model for the molecular double rows where a pair of uprightstanding heterochiral [7] HCA molecules is stabilized by intermolecular hydrogen bond (H-bond) formation between the carboxylic acid moieties. These [7]HCA pairs are then bonded within the row by homochiral $\pi-\pi$ stacking of the aromatic rings. The existence of heterochiral pairs within the rows was confirmed by additional experiments, where after deposition of only the $(M)$ enantiomer no row structures were revealed. ${ }^{11}$ Structures of the single rows (for $\pi-\pi$ stacked $(M)$ enantiomers) and heterochiral double rows are illustrated in Fig. 1(a).

Figure 2 shows NC-AFM images illustrating the time evolution of the racemic [7]HCA row pattern formation for four distinct times in the post-deposition regime. The mean length $\bar{L}$ of rows as a function of time $t$ was extracted from these data with the results shown in Fig. 3. Its evolution reveals two distinct regimes: a ripening regime up to $t \simeq 5500 \mathrm{~s}$ and a saturation regime at later times. In the ripening regime, $\bar{L}$ increases approximately linearly with time.

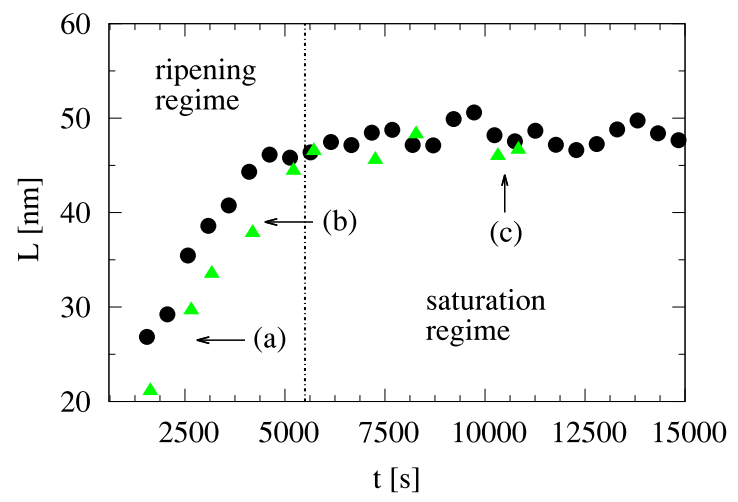

FIG. 3. Time evolution of the mean row length $\bar{L}$ for two different coverages $\Theta=0.03$ (triangles) and $\Theta=0.06$ (circles). The ripening and the saturation regimes are indicated. The crossover between these regimes at $t_{\star} \simeq 5500 \mathrm{~s}$ is marked by a vertical dotted line. The arrows labeled as (a), (b), and (c) refer to mean length evaluations of the three different NC-AFM images in Figs. 2(a)-2(c). 


\section{KINETIC GROWTH MODEL}

An important further experimental finding for the growth of [7]HCA molecules on the calcite (1014) cleavage plane is that ensembles of enantiopure $(M)$-[7]HCA molecules do not form double row structures ${ }^{11}$ in contrast to the observations discussed in Sec. II for the 1:1 racemic mixture of $(M)$ and $(P)$ enantiomers. This implies that double rows are formed by $\mathrm{H}-$ bonding between [7]HCA molecules with different helicities. This conclusion is also supported by DFT calculations of the energies of homo- and hetero-chiral [7]HCA rows in vacuum. ${ }^{9}$ Because $\pi-\pi$ stacking occurs between [7]HCA molecules of the same helicity, it is very likely that single rows are homochiral.

Against this background, we envisage the following picture for the growth kinetics of row formation. After a [7]HCA molecule lands on the substrate, the hydrogen of its carboxylic group and an oxygen atom of a surface carbonate group form a H-bond. As a consequence, if two [7]HCA monomers with different helicity encounter each other on the substrate, they have an unfavorable orientation which impedes the formation of a $\mathrm{H}$-bonded dimer, and hence the nucleation of double rows. However, if a [7]HCA monomer encounters another [7]HCA monomer with the same helicity, the formation of a dimer by $\pi-\pi$ stacking in the [010] direction becomes likely, leading to a nucleation of single rows in favor of double rows. Hence, in early stages of the growth, there is a preferential formation of single rows.

For later stages, the double row formation and the different stabilities of single and double rows become relevant. Single rows grow and shrink by attachment and detachment of [7]HCA monomers to and from the row ends, respectively. By contrast, the H-bonded dimers at the end of double rows are more stable and we can neglect their breakage on the relevant time scale of the experiment (not considering possible Ostwald-type ripening on much longer time scales). The observed behavior of the mean row length in the postdeposition regime, as shown in Fig. 3, could then be caused by a growth of stable double rows at the expense of an average shrinkage of unstable single rows.

To test the reliability of this picture, we employ a rate equation approach with focus on the kinetics of row formation in the submonolayer regime. To keep the treatment simple, an isotropic diffusion of single [7]HCA monomers on the calcite (104) surface is considered, and we do not take explicit account of the two different types of enantiomers. These, however, enter indirectly the modeling by implementing the mechanisms for the shrinkage and growth of rows as described above.

In the specific setup, we introduce the number densities $m_{1}, m_{s}(s \geq 2)$, and $n_{s}^{(\alpha)}(s \geq 2, \alpha=0,1$, or 2$)$, where $m_{1}$ refers to [7]HCA monomers, $m_{s}$ to single rows composed of $s$ [7]HCA molecules, and $n_{s}^{(\alpha)}$ to double rows composed of $s$ H-bonded [7]HCA dimers. The superscript $\alpha$ allows us to distinguish the cases, where either at both ends of a double row no monomer is attached $(\alpha=0)$, where exactly one monomer is attached to one of the two double row ends $(\alpha=1)$, or where one monomer is attached to each of the double row ends $(\alpha=2)$. Single and double rows

are both not mobile and their length (or their type $\alpha$ ) becomes modified by attachment and detachment processes of [7]HCA monomers. These monomers diffuse on the surface with diffusion coefficient $D$. Their capture and attachment to the rows are modelled as in standard rate equation treatments ${ }^{12,13}$ by introducing capture numbers $\sigma_{s}$ and $\sigma_{s}^{(\alpha)}$ for the corresponding single and double rows in connection with bilinear reaction terms $\propto D m_{1} m_{s}$ and $\propto D m_{1} n_{s}^{(\alpha)}$. Also following the standard treatment, the detachment processes of [7]HCA monomers are described by introducing decay rates $k_{s}$ and $k_{s}^{(\alpha)}$ of single rows and double rows of type $\alpha=1$ and $\alpha=2$, respectively.

Five key elementary processes deserve special attention because they are relevant for the interplay of single and double row formation. These are illustrated in Fig. 4 and encompass in panel (a) the nucleation of single rows via formation of dimers in the [010] direction with rate $D \sigma_{1} m_{1}^{2}$ and in panel (b) the nucleation of double rows via formation of H-bonded dimers with rate $D \sigma_{\star} m_{1}^{2}$. The H-bonds in these dimers connect two [7]HCA molecules orthogonal to the [010] growth direction. In Figs. 4(c)-4(e) the evolution of double rows is illustrated in a three-step sequence starting from a H-bonded dimer.

(a)

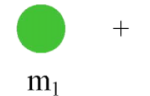

(b)

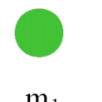

$\mathrm{m}_{1}$

(c)

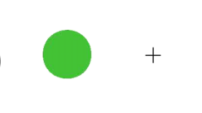

$\mathrm{m}_{1}$

(d)

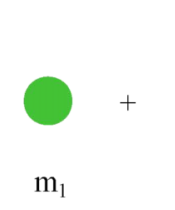

(e)

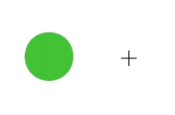

$\mathrm{m}_{1}$
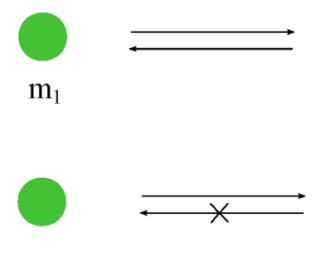

$\mathrm{m}_{1}$
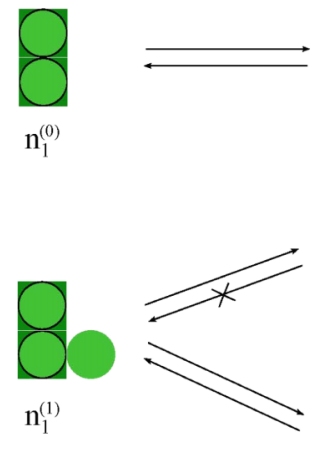

$$
\mathrm{n}_{1}^{(1)}
$$

FIG. 4. Illustration of key elementary processes in the modeling of single and double row formation of racemic [7] HCA molecules on the calcite (104) surface: (a) nucleation of $\pi-\pi$ bonded dimers parallel to the [010] growth direction, (b) nucleation of H-bonded dimers perpendicular to the growth direction, and a multistep sequence describing the evolution of double rows by (c) attachment (detachment) of [7]HCA monomers to (from) double rows of type $\alpha=0(\alpha=1)$, (d) attachment (detachment) of [7]HCA monomers to (from) double rows of type $\alpha=1(\alpha=2)$, and (e) attachment of [7]HCA monomers to double rows of type $\alpha=1$. The crosses on arrows indicate processes that are forbidden. [7] HCA monomers are represented by circles and $\mathrm{H}$-bonded dimers by two connected squares. 
For longer double rows it proceeds analogously. H-bonded dimers cannot break, as indicated by the forbidden processes in Figs. 4(b), 4(d), and 4(e).

The rate equations for the number densities of [7]HCA monomers and single rows of length $s$ for our model read

$$
\begin{aligned}
\frac{d m_{1}}{d t}= & F H\left(t_{\mathrm{dep}}-t\right)-2 D \sigma_{1} m_{1}^{2}-2 D \sigma_{\star} m_{1}^{2}+2 k_{2} m_{2} \\
& -D m_{1} \sum_{s>1} \sigma_{s} m_{s}+\sum_{s>2} k_{s} m_{s} \\
& -D m_{1} \sum_{s \geq 1}\left(\sigma_{s}^{(0)} n_{s}^{(0)}+\sigma_{s}^{(1)} n_{s}^{(1)}+\sigma_{s}^{(2)} n_{s}^{(2)}\right) \\
& +\sum_{s \geq 1}\left(k_{s}^{(1)} n_{s}^{(1)}+k_{s}^{(2)} n_{s}^{(2)}\right), \\
& \frac{d m_{s}}{d t}=D m_{1}\left(\sigma_{s-1} m_{s-1}-\sigma_{s} m_{s}\right) \\
& \quad+k_{s+1} m_{s+1}-k_{s} m_{s}, \quad s \geq 2 .
\end{aligned}
$$

Here, $F H\left(t_{\mathrm{dep}}-t\right)$ describes the deposition of monomers with flux $F$ during the time $t_{\text {dep }}$ of deposition, where $H($.$) is$ the Heaviside jump function $[H(x)=1$ for $x \geq 1$ and zero otherwise]. The three other terms in the first line on the right hand side of Eq. (1) describe the loss of monomers due to nucleation of single rows (formation of $\pi$ - $\pi$-bonded dimers), the loss of monomers due to the nucleation of double rows (formation of H-bonded dimers), and the gain of monomers due to dissociation of $\pi$ - $\pi$-bonded dimers. The remaining terms on the right hand side of Eq. (1), as well as on the right hand side of the set of Eq. (2), describe the attachment and detachment of monomers to single and double rows.

The number densities of double rows of type $\alpha$ evolve according to the rate equations

$$
\begin{gathered}
\frac{d n_{s}^{(0)}}{d t}=-D m_{1} \sigma_{s}^{(0)} n_{s}^{(0)}+k_{s}^{(1)} n_{s}^{(1)} \\
+ \begin{cases}D \sigma_{\star} m_{1}^{2}, & s=1, \\
\frac{1}{2} D \sigma_{s-1}^{(1)} m_{1} n_{s-1}^{(1)}, & s \geq 2,\end{cases} \\
\frac{d n_{s}^{(1)}}{d t}=D m_{1}\left(\sigma_{s}^{(0)} n_{s}^{(0)}-\sigma_{s}^{(1)} n_{s}^{(1)}\right)+k_{s}^{(2)} n_{s}^{(2)}-k_{s}^{(1)} n_{s}^{(1)}
\end{gathered}
$$

The case distinction in the set of Eq. (3) takes into account that for $s=1$, there is a gain term due to nucleation of $\mathrm{H}$-bonded dimers, while for $s \geq 2$ there is a gain term, because monomers can attach to a double row of type $\alpha=1$ of length $s-1$ and form a H-bonded dimer at one end [see the upper reaction path in Fig. 4(d)]. The factor $1 / 2$ in front of the corresponding rate $D \sigma_{s-1}^{(1)} m_{1} n_{s-1}^{(1)}$ arises from the fact that a H-bonded dimer forms only if a monomer attaches to that end of the respective double row where a monomer was already attached to. An attachment to the other end would give a gain contribution to the evolution of $n_{s-1}^{(2)}$, as described by the first term on the right hand side of Eq. (5). In the set of Eq. (4), the case distinction takes into account that an increase of the density of double rows of type $\alpha=1$ by attachment of monomers to double rows of type $\alpha=2$ [cf. Fig. 4(e)] is possible only for $s \geq 2$. The remaining terms in Eqs. (3)-(5) describe attachments and detachments of monomers to the different types of double rows.

Equations (1)-(5) constitute a complete set of rate equations and their numerical solution in principle allows one to predict not only the time evolution of the mean row length, but also the time evolution of the full length distributions of single and double rows. However, a difficult problem here is the correct treatment of the capture numbers $\sigma_{s}, \sigma_{s}^{(\alpha)}$ and of the decay rates $\kappa_{s}, \kappa_{s}^{(\alpha)}$. Even for the simplest cases of submonolayer growth of atomic clusters, this is an unsolved problem. The capture numbers and decay rates in general have rather complicated dependencies on $s$, the total coverage, and the $D / F$ ratio ${ }^{36,37}$ If one is interested just in mean cluster sizes (first moments of the cluster size distribution), a self-consistent calculation can provide good results. In this calculation, the capture numbers and decay rates follow from a solution of a diffusion equation for the monomers with appropriate boundary conditions, in which the cluster densities from the rate equations enter collectively as an effective absorption length. ${ }^{34,35}$ However, as recently shown by some of the authors, ${ }^{38}$ a generalization of this self-consistent theory to systems with different types of components, as reflected in the $\pi-\pi$ and $\mathrm{H}$-bonded dimers in the situation considered here, requires rather elaborate treatments involving pair-distribution functions. Moreover, the one-dimensional chain-structure of the islands needs to be taken into account when calculating the diffusion field of the ad-molecules around the chains. For our purpose of getting basic insight into the interplay of single and double row formation, we do not attempt here to go in such detail, but will use simple expressions for the capture numbers and decay rates, similar as it was done in the past to understand certain aspects in atomic cluster growth.

Specifically we set $\sigma_{s}=\sigma_{s}^{(\alpha)}=\sigma_{0} s^{1 / 2}$ with $\sigma_{0}=1$, $k_{s}=k_{s}^{(1)}=k_{s}^{(2)} / 2=D \exp \left(-\beta U_{\pi}\right)$, where $\beta=1 / k_{\mathrm{B}} T$ is the inverse thermal energy and $U_{\pi}$ is the activation energy for the detachment of a $\pi-\pi$ bonded [7]HCA monomer from a row (and for the dissociation of a $\pi-\pi$ bonded dimer). To take into account that the nucleation of a H-bonded dimer is impeded compared to the nucleation of a $\pi-\pi$ bonded dimer, we assume that it requires an additional activation energy $U_{\star}$ and accordingly set $\sigma_{\star}=\sigma_{0} \exp \left(-\beta U_{\star}\right)$. The diffusion of the [7]HCA monomers is thermally activated with the diffusion barrier $U_{D}$, i.e., $D=D_{0} \exp \left(-\beta U_{D}\right)$.

It remains to specify the values of the activation energies $U_{\pi}, U_{D}$, and $U_{\star}$, and of the prefactor $D_{0}$. The energy $U_{\pi}$ can be roughly estimated from the binding energy of a $\pi-\pi$ bonded dimer obtained from DFT calculations, ${ }^{9}$ giving $U_{\pi}=0.175 \mathrm{eV}$ as a reasonable value. For the diffusion barrier we take $U_{D}=0.52 \mathrm{eV}$, which lies in the range estimated from experiments. ${ }^{9}$ The prefactor $D_{0}$ is set to $D_{0}=10^{10} \mathrm{~nm}^{2} \mathrm{~s}^{-1}$, which corresponds to a typical (molecular) attempt frequency 
(a)

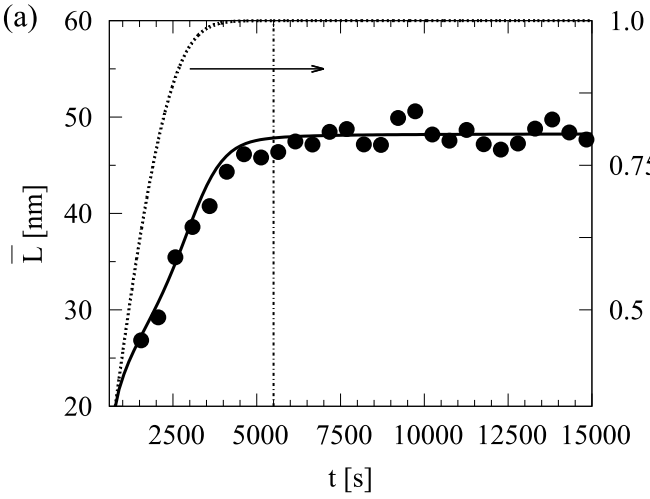

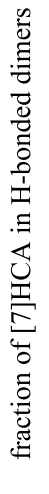

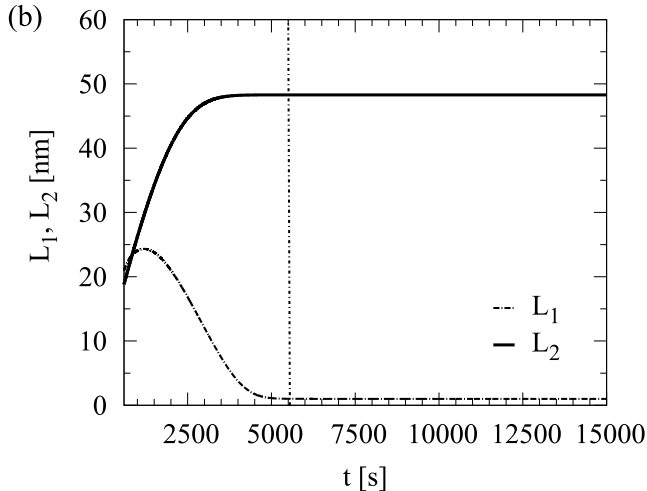

FIG. 5. Time evolution of (a) the mean length $\bar{L}$ (black solid line) and fraction of H-bonded dimers (dotted line), and (b) the mean lengths $L_{1}$ and $L_{2}$ of single and double rows as predicted by the mean-field rate equations (1)-(5). In (a) the experimental data (circles) from Fig. 3 are replotted for comparison. The deposition time was $t_{\mathrm{dep}}=600 \mathrm{~s}$ (corresponding to a coverage $\Theta=0.06$ ), and the data were simulated at room temperature $T=300 \mathrm{~K}$ with energetic parameters $U_{D}=0.52 \mathrm{eV}, U_{\pi}=0.175 \mathrm{eV}$, and $U_{\star}=0.118 \mathrm{eV}$. The vertical dashed-dotted lines indicate the crossover from the ripening to the saturation regime.

of $4 \times 10^{10} \mathrm{~s}^{-1}$, if considering a mean jump length of about $0.5 \mathrm{~nm}$ for a diffusion step of a [7]HCA monomer, corresponding to the lattice spacing in the [010] direction on the calcite (104) surface. With respect to the energy $U_{\star}$, we leave it as the only adjustable parameter to recover the observed behavior of the mean row length in Fig. 3.

For the total number density

$$
N=\sum_{s=1}^{\infty}\left[s m_{s}+\sum_{\alpha=0}^{2}(2 s+\alpha) n_{s}^{(\alpha)}\right]
$$

of [7]HCA molecules, one obtains $d N / d t=F H\left(t_{\text {dep }}-t\right)$ from Eqs. (1)-(5), as required by mass conservation. Accordingly, after the deposition time $t_{\text {dep }}=600 \mathrm{~s}, N$ attains the constant value $F t_{\text {dep }} \simeq 0.05\left(F \simeq 8.3 \times 10^{-5} \mathrm{~s}^{-1}\right.$, cf. Sec. II $)$.

The mean lengths $L_{1}$ and $L_{2}$ of single and double rows are given by

$$
\begin{aligned}
& L_{1}=\frac{d}{N_{\mathrm{r} 1}} \sum_{s=1}^{\infty} m_{s} s, \\
& L_{2}=\frac{d}{N_{\mathrm{r} 2}} \sum_{s=1}^{\infty} \sum_{\alpha=0}^{2} n_{s}^{(\alpha)} s,
\end{aligned}
$$

where $N_{\mathrm{r} 1}=\sum_{s=1}^{\infty} m_{s}$ and $N_{\mathrm{r} 2}=\sum_{s=1}^{\infty} \sum_{\alpha=0}^{2} n_{s}^{(\alpha)}$ are the number densities of single and double rows, respectively, and $d=1 \mathrm{~nm}$ is the distance of two $\pi-\pi$ bonded [7]HCA molecules in the rows. ${ }^{9}$ The mean length of all rows is

$$
\bar{L}=\frac{\left(N_{\mathrm{r} 1} L_{1}+N_{\mathrm{r} 2} L_{2}\right)}{N_{\mathrm{r} 1}+N_{\mathrm{r} 2}} .
$$

Figure 5(a) shows the time evolution of $\bar{L}$ as predicted by the modeling (solid line) in comparison with the experimental findings (circles), where we adjusted $U_{\star}$ to give the crossover to the saturation at time $t_{\star} \simeq 5500 \mathrm{~s}$. For the data shown, the value $U_{\star}=0.118 \mathrm{eV}$ was used. As can be seen from the figure, the kinetic growth model describes well the experimentally observed time evolution, and the value of $47 \mathrm{~nm}$ for $\bar{L}$ in the saturation regime is reproduced within the experimental error. It is interesting to note that the theoretical results do not show a strict linear increase of $\bar{L}$ with time in the ripening regime. The deviations from a linear behavior seem to be reflected by the experimental data although the accuracy is not sufficient to really confirm the predicted deviations from a linear increase. From the behavior of the fraction $(2 / N) \sum_{s=1}^{\infty} \sum_{\alpha=0}^{2} s n_{s}^{(\alpha)}$ of [7]HCA molecules that belong to H-bonded dimers, shown as dotted line in Fig. 5(a), we can conclude that almost all single rows have disappeared at the onset of the saturation regime.

The growth of double rows at the expense of single rows is reflected also in the behavior of their mean lengths $L_{2}$ and $L_{1}$ shown in Fig. 5(b). The length $L_{1}$ runs through a maximum at $t \simeq 1500 \mathrm{~s}$, where attachment and detachment of [7]HCA molecules to the single rows of various lengths become balanced with respect to a change of $L_{1}$. Thereafter, detachment processes become dominant and $L_{1}$ decreases until almost all single rows disappeared at $t \simeq t_{\star}$. In contrast, the mean length $L_{2}$ monotonically increases until time $t_{\star}$ because [7]HCA molecules detached from single rows can form stable $\mathrm{H}$-bonded molecules at the ends of double rows. There are also a few [7]HCA molecules detaching from double rows of type $\alpha=1$ and $\alpha=2$, cf. Fig. 4, but these are not relevant for a change of $L_{2}$ because a double row of type $\alpha=1$ or $\alpha=2$ can at most decay to a corresponding double row of type $\alpha=0$, leaving its length effectively unchanged. In fact, in Eq. (8) we defined the length of the double rows with respect to the number of $\mathrm{H}$-bonded [7] HCA molecules in these rows.

\section{CONCLUSIONS}

The self-assembly of racemic [7]HCA molecules on calcite (104) into double stripes is to date one of the rare examples of molecular wire formation on insulating surfaces. In this work, we gave strong support that hydrogen bonding between distinct [7]HCA enantiomers orthogonal to the [010] growth direction is the key mechanism for the wire formation. This hydrogen bonding between heterochiral [7]HCA molecules requires an extra activation energy. While [7]HCA molecules of the same helicity self-assemble into single rows by $\pi-\pi$ stacking, these single rows are not stable at room temperature. By overcoming an activation barrier associated with the breakage of the $\mathrm{H}$-bond between the [7]HCA's carboxylic group and the substrate's carbonate 
ion, two [7]HCA molecules of different helicity can form a $\mathrm{H}$-bonded dimer. These $\mathrm{H}$-bonded dimers act as nuclei for the formation of the more stable double rows, in which the [7]HCA molecules are held together by both $\pi-\pi$ stacking along and intermolecular $\mathrm{H}$-bonds perpendicular to the rows.

Following concepts of the standard rate equation theory, we developed a rate equation approach to model the feasible mechanism of the double-stripe formation. Key elementary processes in this approach are the nucleation of $\pi-\pi$ bonded dimers in the [010] growth direction, the nucleation of $\mathrm{H}$ bonded dimers perpendicular to the row growth direction, and the attachment and detachment of monomers to single and double rows. Parameters entering the rates for the elementary processes were estimated from binding energies and DFT calculations. Both experimentally and within the model, we found two sequential but distinct growth regimes in the time evolution of the mean molecular row length $\bar{L}(t)$. While $\bar{L}(t)$ increases with time in the first regime, a saturated behavior is observed second, where $\bar{L}(t)$ remains constant. We identified this behavior as a transition between a ripening and a saturation regime, in agreement with experimental observation. In the saturation regime, the system converges to a phase of exclusive heterochiral molecular double rows, while the ripening starts from a coexistence of homochiral single and heterochiral double rows. Fitting the model to the experimental data yielded a value of about $120 \mathrm{meV}$ for the extra activation energy needed for intermolecular H-bond formation.

From a general point of view, our work gives a further example for the power of rate equation theories in describing aspects of self-organized structure formation on surfaces in the submonolayer growth regime. Originally developed to describe atomic cluster growth, the theory seems to be well suited also for describing molecular cluster growth after proper adaptation. One-dimensional molecular row formation is a particular case and it will be interesting to see in the future how far refinements of the theory, as the self-consistent treatment of capture numbers under consideration of different types of molecular species, will allow one to describe further details of the experimental observations.

\section{ACKNOWLEDGMENTS}

We are grateful to M. Körner for valuable discussions, and M.E. thanks D. R. T. Zahn for helpful comments on the growth of wire-like structures on free terraces. This work has been supported by the German Research Foundation (DFG) through the Emmy Noether-program (Grant Nos. KU 1980/1-2 and KU 1980/1-3).
${ }^{1}$ A. Nitzan and M. A. Ratner, Science 300, 1384 (2003).

${ }^{2}$ Y. V. Nazarov and Y. M. Blanter, Quantum Transport (Cambridge University Press, Cambridge, 2009).

${ }^{3}$ J. C. Cuevas and E. Scheer, Molecular Electronics: An Introduction to Theory and Experiment (World Scientific, Singapore, 2010).

${ }^{4}$ S. Kubatkin, A. Danilov, M. Hjort, J. Cornil, J. Bredas, N. Stuhr-Hansen, P. Hedegard, and T. Bjornholm, Nature 425, 698 (2003).

${ }^{5}$ N. Wang, Y. Cai, and R. Q. Zhang, Mater. Sci. Eng., R 60, 1 (2008).

${ }^{6}$ P. Rahe, M. Kittelmann, J. L. Neff, M. Nimmerich, M. Reichling, P. Maass, and A. Kühnle, Adv. Mater. 25, 3948 (2013).

${ }^{7}$ Y. Suzuki, M. Hietschold, and D. R. T. Zahn, Appl. Surf. Sci. 252, 5449 (2006).

${ }^{8}$ J. Neff, H. Söngen, R. Bechstein, P. Maass, and A. Kühnle, J. Phys. Chem. C 119, 24927 (2015).

${ }^{9}$ P. Rahe, M. Nimmrich, A. Greuling, J. Schütte, I. G. Stará, J. Rybáček, G. Huerta-Angeles, I. Starý, M. Rohlfing, and A. Kühnle, J. Phys. Chem. C 114, 1547 (2011).

${ }^{10}$ J. Rybáček, G. Huerta-Angeles, A. Kollárovič, I. G. Stará, I. Starý, P. Rahe, M. Nimmrich, and A. Kühnle, Eur. J. Org. Chem. 2011, 853 (2011).

${ }^{11}$ C. M. Hauke, P. Rahe, M. Nimmrich, J. Schütte, M. Kittelmann, I. G. Stará, I. Starý, J. Rybáček, and A. Kühnle, J. Phys. Chem. C 116, 4637 (2012).

${ }^{12}$ J. A. Venables, Philos. Mag. 27, 697 (1973).

${ }^{13}$ J. A. Venables, G. D. T. Spiller, and M. Hanbücken, Rep. Prog. Phys. 47, 399 (1984).

${ }^{14}$ L. Tröger, J. Schütte, F. Ostendorf, A. Kühnle, and M. Reichling, Rev. Sci. Instrum. 80, 063703 (2009).

${ }^{15}$ P. Rahe, J. Schütte, and A. Kühnle, J. Phys.: Condens. Matter 24, 084006 (2012).

${ }^{16}$ One molecule covers $1.2 \mathrm{~nm}^{2}$ as determined from the $(2 \times 3)$ superstructure for $(M)$-[7]HCA, see also Ref. 11.

${ }^{17}$ T. R. Albrecht, P. Grütter, D. Horne, and D. Rugar, J. Appl. Phys. 69, 668 (1991).

${ }^{18}$ F. J. Giessibl, Phys. Rev. B 56, 16010 (1997).

${ }^{19}$ H. Brune, Surf. Sci. Rep. 31, 121 (1998).

${ }^{20} \mathrm{~T}$. Michely and J. Krug, Islands, Mound and Atoms (Springer, Berlin, 2004).

${ }^{21}$ J. W. Evans, P. A. Thiel, and M. C. Bartelt, Surf. Sci. Rep. 61, 1 (2006).

${ }^{22}$ M. Einax, W. Dieterich, and P. Maass, Rev. Mod. Phys. 85, 921 (2013).

${ }^{23}$ H. Brune, G. S. Bales, J. Jacobsen, C. Boragno, and K. Kern, Phys. Rev. B 60, 5991 (1999).

${ }^{24}$ B. Müller, L. Nedelmann, B. Fischer, H. Brune, and K. Kern, Phys. Rev. B 54, 17858 (1996).

${ }^{25}$ G. Richter and T. Wagner, J. Appl. Phys. 98, 094908 (2005).

${ }^{26} \mathrm{G}$. Hlawacek, P. Puschnig, P. Frank, A. Winkler, C. Ambrosch-Draxl, and C. Teichert, Science 321, 108 (2008).

${ }^{27}$ T. Potocar, S. Lorbek, D. Nabok, Q. Shen, L. Tumbek, G. Hlawacek, P. Puschnig, C. Ambrosch-Draxl, C. Teichert, and A. Winkler, Phys. Rev. B 83, 075423 (2011).

${ }^{28}$ A. Winkler and L. Tumbek, Phys. Chem. Lett. 4, 4080 (2013).

${ }^{29}$ M. Körner, F. Loske, M. Einax, A. Kühnle, M. Reichling, and P. Maass, Phys. Rev. Lett. 107, 016101 (2011).

${ }^{30}$ A. Zangwill and D. D. Vvedensky, Nano Lett. 11, 2092 (2011).

${ }^{31}$ M. Einax, S. Ziehm, W. Dieterich, and P. Maass, Phys. Rev. Lett. 99, 016106 (2007).

${ }^{32}$ W. Dieterich, M. Einax, and P. Maass, Eur. Phys. J. 161, 151 (2008).

${ }^{33}$ M. Einax, W. Dieterich, and P. Maass, J. Appl. Phys. 105, 054312 (2009).

${ }^{34}$ G. S. Bales and D. C. Chrzan, Phys. Rev. B 50, 6057 (1994).

${ }^{35}$ G. S. Bales and A. Zangwill, Phys. Rev. B 55, R1973 (1997).

${ }^{36}$ M. Körner, M. Einax, and P. Maass, Phys. Rev. B 82, 201401(R) (2010).

${ }^{37}$ M. Körner, M. Einax, and P. Maass, Phys. Rev. B 86, 085403 (2012).

${ }^{38}$ M. Einax, P. Maass, and W. Dieterich, Phys. Rev. B 90, 035441 (2014). 